\title{
Porous Graphene Oxide/Diboronic Acid Materials: Structure and Hydrogen sorption.
}

Guillaume Mercier $^{\mathrm{a}}$, Alexey Klechikov ${ }^{\mathrm{a}}$, Mattias Hedenström ${ }^{b}$, Dan Johnels ${ }^{b}$, Igor A. Baburin ${ }^{c}$, Gotthard Seifert, ${ }^{c}$ Roman Mysyk, ${ }^{d}$ Alexandr V. Talyzin ${ }^{\mathrm{a}, *}$

${ }^{a}$ Department of Physics, Umeå University, SE-901 87 Umeå, Sweden.

${ }^{\mathrm{b}}$ Department of Chemistry, Umeå University, SE-901 87 Umeå, Sweden.

c Technische Universität Dresden, Theoretische Chemie, Bergstraße 66b, 01062 Dresden, Germany

${ }^{\mathrm{d}}$ CIC EnergiGUNE, Arabako Teknologi Parkea, Minano 01510, Spain.

*Corresponding author e-mail: alexandr.talyzin@ physics.umu.se

\section{Additional nitrogen sorption data.}
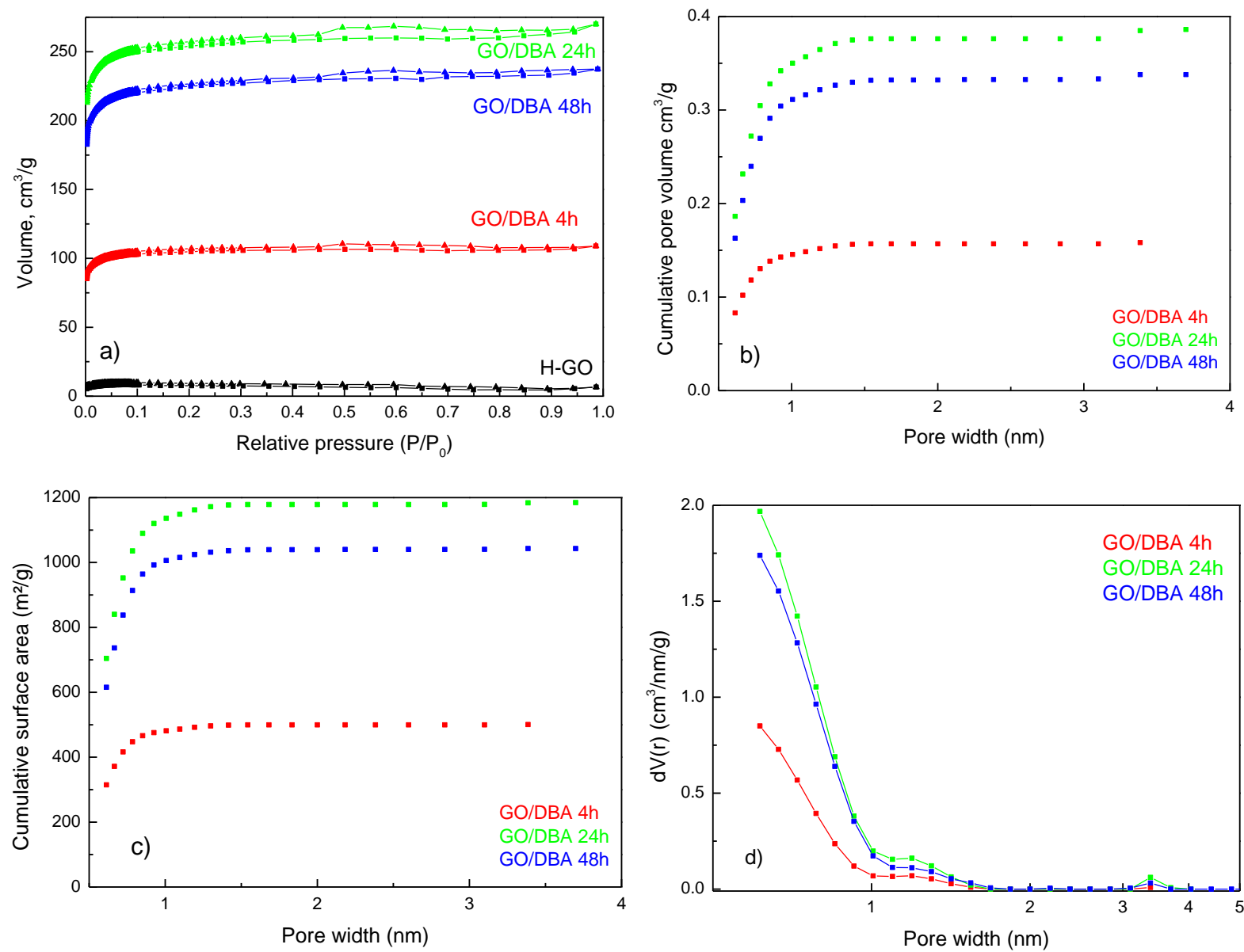

Figure 1S a) Examples of nitrogen sorption isotherms measured on GO/DBA samples prepared using a duration of GO-DBA reaction time of 4-48 hours and properties simulated using the QSDFT slit pore model b) pore size distribution, c) cumulative surface area d) cumulative pore volume. 
2.Details of FTIR data.

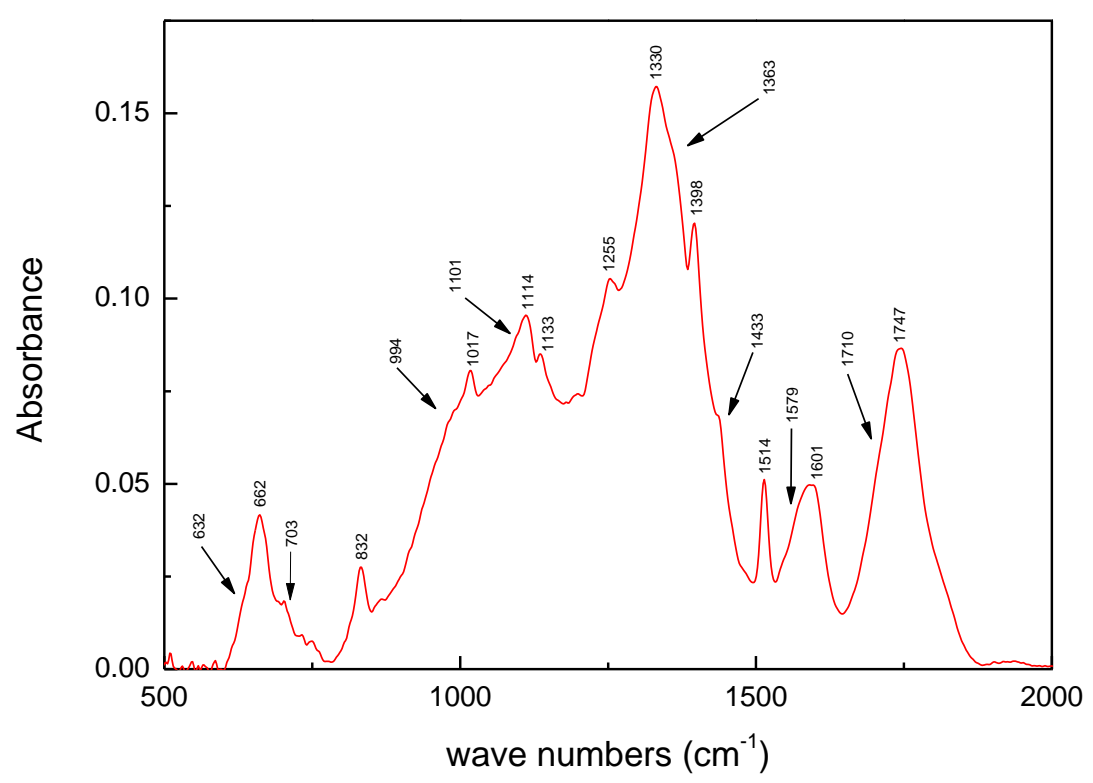

Figure 2S Example of typical FTIR spectra of GO/DBA sample with peak positions. 


\begin{tabular}{|c|c|c|c|c|c|c|c|c|}
\hline $\begin{array}{c}\text { Graphite } \\
\text { oxide }\end{array}$ & DBA & GO/DBA & $\mathrm{GO} / \mathrm{DBA}$ & $\mathrm{GO} / \mathrm{DBA}$ & GO/DBA & GO/DBA & GO/DBA & GO/DBA \\
\hline $\begin{array}{c}\mathrm{GO}: \mathrm{DBA} \\
\text { ratio }\end{array}$ & & $1: 1$ & $1: 3$ & $1: 3$ & $1: 3$ & $1: 6$ & $1: 3$ & $1: 3$ \\
\hline \multirow[t]{5}{*}{$\begin{array}{l}\text { Reaction } \\
\text { time }\end{array}$} & & $96 \mathrm{~h}$ & $24 \mathrm{~h}$ & $48 \mathrm{~h}$ & $24 \mathrm{~h}$ & $24 \mathrm{~h}$ & $4 \mathrm{~h}$ & $24 \mathrm{~h}$ \\
\hline & 625 (sh) & & 633 & 634 & 632 & 630 & 636 & 633 \\
\hline & $651(\mathrm{sp})$ & & 662 & 655 & 662 & 661 & 660 & 663 \\
\hline & 669 (sp) & & 703 & 703 & 703 & 703 & 704 & 701 \\
\hline & & & & 755 & & & & 752 \\
\hline \multicolumn{9}{|l|}{796 (sh) } \\
\hline 813 (sh) & 809 (sp) & & & & & & & \\
\hline 839 (br) & 836 & & 831 & 835 & 832 & 828 & 832 & 833 \\
\hline \multicolumn{9}{|l|}{846 (sh) } \\
\hline & 989 & & 987 & 987 & 994 & & 982 & 986 \\
\hline & 1010 (sp) & & 1012 & 1015 & 1017 & 1018 & 1016 & 1016 \\
\hline & 1035 (sp) & & 1038 & & & & & \\
\hline 1053 (br) & & & 1067 & & & & & \\
\hline \multirow[t]{4}{*}{1089 (sh) } & 1104 & 1094 & 1092 & 1092 & 1101 & 1103 & & \\
\hline & & & 1111 & 1111 & 1114 & 1116 & 1111 & 1111 \\
\hline & 1130 (sp) & & 1137 & 1142 & 1133 & 1139 & 1139 & 1141 \\
\hline & & & 1150 & & & & & \\
\hline 1173 (sh) & 1169 (sp) & 1163 & 1168 & & & & & \\
\hline \multirow[t]{3}{*}{1227 (br) } & & 1240 & & & & & & \\
\hline & 1251 (sh) & & 1252 & 1255 & 1255 & 1256 & 1252 & 1251 \\
\hline & 1261 (sp) & & & & & & & \\
\hline 1296 (sh) & 1300 (sh) & 1304 & & & & & & \\
\hline \multirow[t]{3}{*}{1362 (br) } & 1345 (br) & 1351 & 1335 & 1331 & 1330 & 1328 & 1334 & 1333 \\
\hline & 1379 (sh) & & 1366 & 1634 & 1363 & 1366 & 1370 & 1367 \\
\hline & 1399 (sp) & & 1398 & 1398 & 1398 & 1400 & 1398 & 1399 \\
\hline \multirow[t]{3}{*}{1406 (br) } & 1422 (sh) & 1439 & & 1430 & 1433 & 1432 & & \\
\hline & & 1484 & 1488 & & & & & \\
\hline & 1515 (sp) & 1517 & 1514 & 1512 & 1514 & 1514 & 1514 & 1514 \\
\hline 1569 (sh) & & 1553 & 1568 & 1566 & 1579 & & 1576 & 1574 \\
\hline
\end{tabular}




\begin{tabular}{|c|c|c|c|c|c|c|c|c|}
\hline & 1585 (sh) & 1588 & 1584 & & & & & \\
\hline \multirow[t]{3}{*}{1611 (br) } & 1614 (sh) & & 1603 & 1597 & 1601 & 1603 & 1603 & 1602 \\
\hline & & 1656 & & & & & & \\
\hline & 1700 (sp) & & 1703 & 1695 & 1710 & 1706 & 1709 & 1719 \\
\hline \multirow[t]{3}{*}{1744 (br) } & & 1750 & 1750 & 1744 & 1747 & 1747 & 1748 & 1753 \\
\hline & 1782 (br) & & & & & & & \\
\hline & 1801 (br) & & 1803 & & & 1787 & 1796 & 1798 \\
\hline \multirow[t]{19}{*}{1832 (sh) } & 1838 (br) & & & & & & & \\
\hline & 1848 (sh) & & & & & & & \\
\hline & 1944 (sp) & & & & & & & \\
\hline & 2019 (br) & & & & & & & \\
\hline & 2037 (br) & & & & & & & \\
\hline & 2099 (br) & & & & & & & \\
\hline & 2127 (br) & & & & & & & \\
\hline & 2172 (br) & & & & & & & \\
\hline & 2261 (sh) & & & & & & & \\
\hline & 2281 (br) & & & & & & & \\
\hline & 2307 (br) & & & & & & & \\
\hline & 2406 (br) & & & & & & & \\
\hline & & & & & 2951 & 2949 & 2953 & 2952 \\
\hline & 3025 (sp) & & & & & 3021 & & 3024 \\
\hline & 3043 (sp) & & & & & & & \\
\hline & 3074 (sp) & & & & 3071 & 3072 & 3071 & 3068 \\
\hline & 3217 (sh) & & & & & & & \\
\hline & 3296 (br) & & & & & 3323 & & \\
\hline & 3422 (br) & & & & & 3494 & & \\
\hline
\end{tabular}

(sp): sharp

(sh): shoulder

(br): broad

Table 1S. Results of detailed fitting of FTIR spectra with peak positions found for several GO/DBA samples . 


\section{Additional XPS data.}

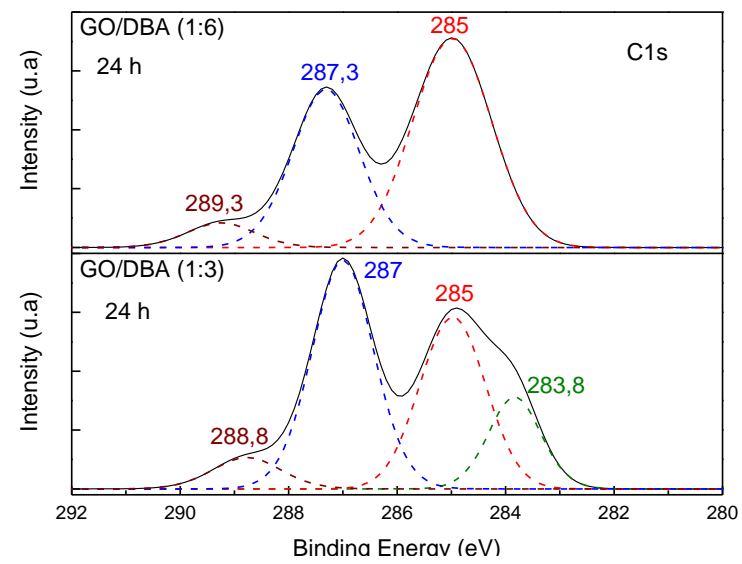

a)

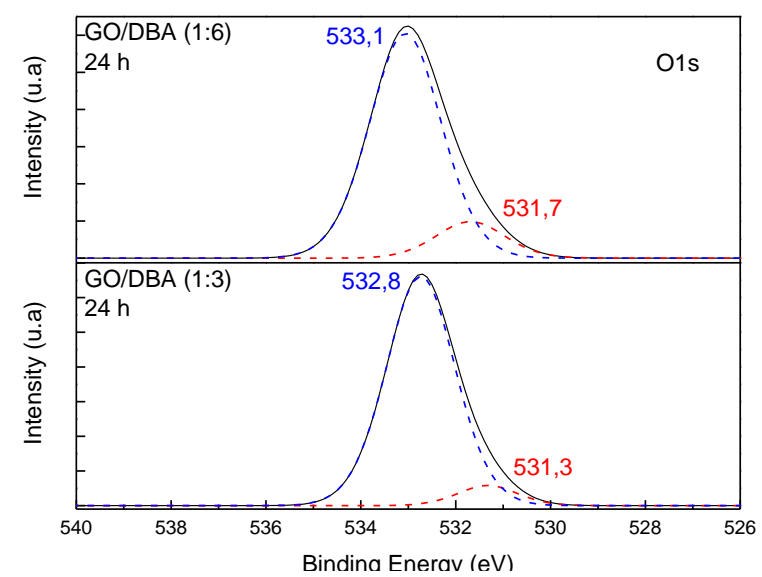

b)

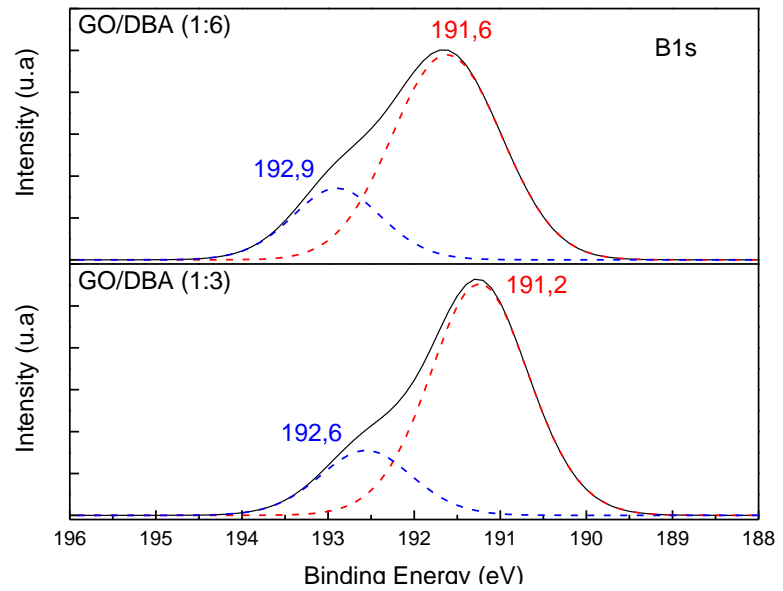

c)

Figure 3S. XPS spectra recorded from samples with initial load GO:DBA 1:6 (B-2.95\%) and 1:3 (B-2.61\%) and reaction time 24 hours. 


\section{Additional hydrogen sorption isotherms.}

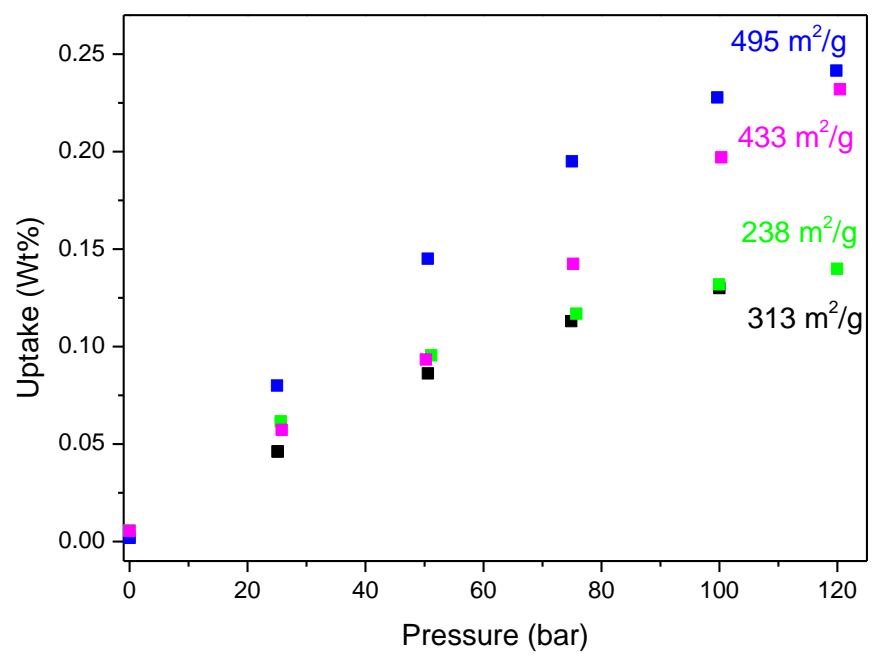

Figure 4S. Hydrogen sorption isotherms recorded from GO/DBA samples with different SSA value using gravimetric method (manual dosing) at $293 \mathrm{~K}$.

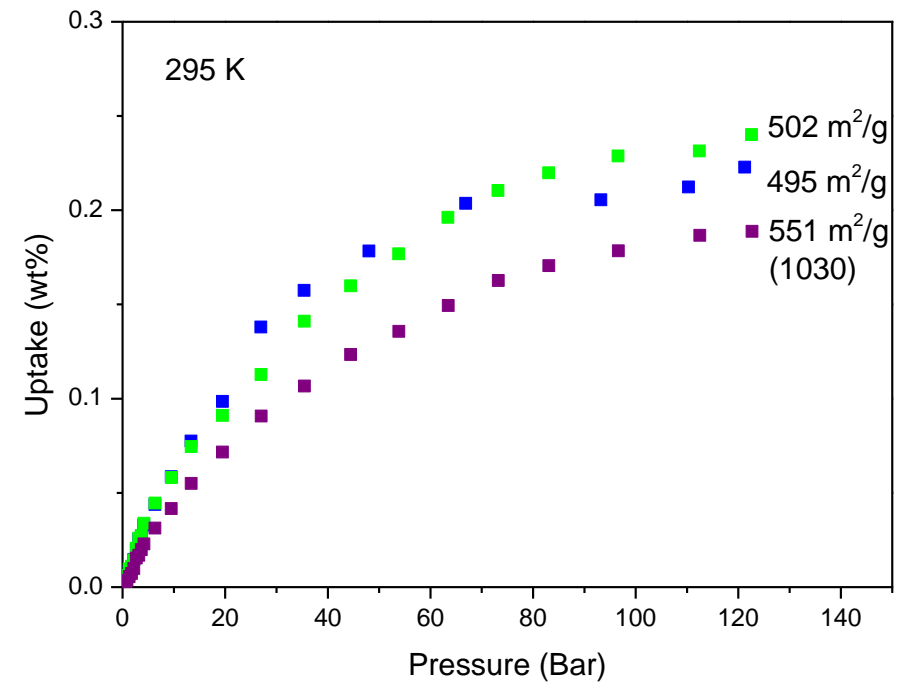

Figure 5S Hydrogen sorption isotherms recorded from GO/DBA samples with different SSA values using volumetric method at $295 \mathrm{~K}$. SSA values measured prior to $\mathrm{H}_{2}$ uptake measurements are provided in () when available. 


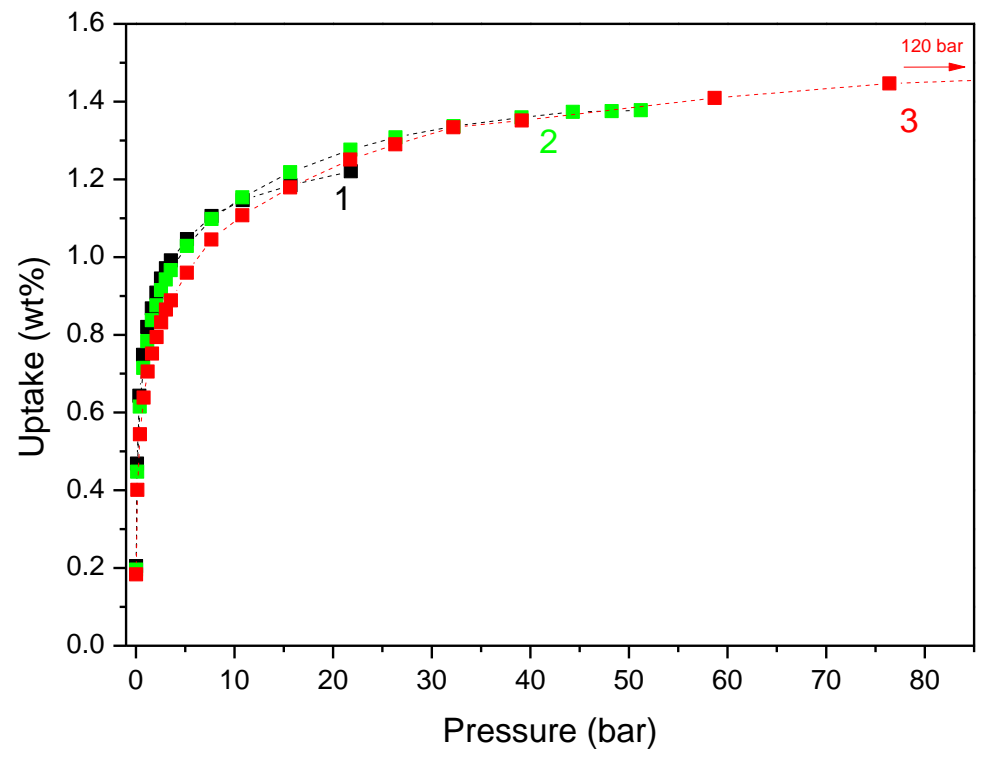

Figure 6S. Hydrogen sorption isotherms recorded from GO/DBA sample at 77K. The sample was first degassed at $120^{\circ} \mathrm{C}$ for 24 hours, isotherm 1 was recorded up to $\sim 20$ bar (1), then the sample was degassed for 24 hours at $77 \mathrm{~K}$ and isotherm recorded up to $\sim 50$ bar (2) and after 24 hours degassing at $77 \mathrm{~K}$ another isotherm was recorded up to $\sim 120$ bar (3). On forth step the sample was carefully degassed at ambient temperature for 24 hours and isotherm recorded at 77 $\mathrm{K}$ up to 20 bar once again (not shown). This isotherm showed no decrease of hydrogen uptake compared to isotherm recorded on step 1. Therefore, no obvious signs of pore structure collapse can be detected for pressure range 20-120 bar. However, the BET surface area was found to decrease from $540 \mathrm{~m}^{2} / \mathrm{g}$ to $430 \mathrm{~m}^{2} / \mathrm{g}$. 